\title{
Simulation of a Miniature, Low-Power Time-of-Flight Mass Spectrometer for In Situ Analysis of Planetary Atmospheres
}

Todd. T. King ${ }^{* a}$, Stephanie A. Getty ${ }^{a}$, Patrick A. Roman ${ }^{b}$, Federico A. Herrero ${ }^{b}$, Hollis H. Jones ${ }^{b}$, Duncan M. Kahle ${ }^{b}$, Bernard Lynch ${ }^{b}$, George Suárez ${ }^{c}$, William B. Brinckerhoff ${ }^{d}$, Paul R. Mahaffy ${ }^{d}$ ${ }^{2}$ NASA Goddard Space Flight Center, Materials Engineering Branch/541, Greenbelt, MD 20771;

bNASA Goddard Space Flight Center, Detector Systems Branch/553, Greenbelt, MD 20771;

${ }^{c}$ NASA Goddard Space Flight Center, Microelectronics Branch/564, Greenbelt, MD 20771;

${ }^{d}$ NASA Goddard Space Flight Center, Atmospheric Experiments Lab/699, Greenbelt, MD 20771;

\begin{abstract}
We are implementing nano- and micro-technologies to develop a miniaturized electron impact ionization mass spectrometer for planetary science. Microfabrication technology is used to fabricate the ion and electron optics, and a carbon nanotube (CNT) cathode is used to generate the ionizing electron beam. Future NASA planetary science missions demand miniaturized, low power mass spectrometers that exhibit high resolution and sensitivity to search for evidence of past and present habitability on the surface and in the atmosphere of priority targets such as Mars, Titan, Enceladus, Venus, Europa, and short-period comets. Toward this objective, we are developing a miniature, high resolution reflectron time-of-flight mass spectrometer (Mini TOF-MS) that features a low-power CNT field emission electron impact ionization source and microfabricated ion optics and reflectron mass analyzer in a parallel-plate geometry that is scalable. Charged particle electrodynamic modeling (SIMION 8.0.4) is employed to guide the iterative design of electron and ion optic components and to characterize the overall performance of the Mini TOF-MS device via simulation. Miniature $\left(<1000 \mathrm{~cm}^{3}\right)$ TOF-MS designs (ion source, mass analyzer, detector only) demonstrate simulated mass resolutions $>600$ at sensitivity levels on the order of $10^{-3} \mathrm{cps} / \mathrm{molecule}_{2} / \mathrm{cc}$ while consuming $1.3 \mathrm{~W}$ of power and are comparable to current spaceflight mass spectrometers. Higher performance designs have also been simulated and indicate mass resolutions $\sim 1000$, though at the expense of sensitivity and instrument volume.
\end{abstract}

Keywords: miniature time-of-flight mass spectrometer, simulation

\section{INTRODUCTION}

The importance of NASA astrobiological and planetary science missions to high priority targets such as Mars, Titan, Enceladus, Europa, Venus, the Moon and short-period comets is emphasized in NASA's 2006 Strategic Plan' and Vision for Space Exploration ${ }^{2}$. Future missions to these challenging destinations will be driven by science objectives that require in situ measurements of volatiles to advance scientific knowledge of the origin and history of volatiles in the solar system, the potential for life elsewhere, and the availability of resources as humans explore space. Mass spectrometers that exhibit high mass resolution and sensitivity across a wide mass range will be critical for detailed isotopic measurements of volatile species to constrain the origin and processing of light elements $(\mathrm{C}, \mathrm{H}, \mathrm{O}, \mathrm{N}$, and $\mathrm{S})$, the noble gases, and prebiotic organic compounds. In situ chemical and isotopic measurements will be a core element of many if not all of the missions targeted for the next decade and beyond. These science objectives and associated mass spectrometer requirements are listed in Table 1 along with the targeted NASA missions.

Current state-of-the-art space flight mass spectrometers, such as the Sample Analysis at Mars (SAM) Quadrupole Mass Spectrometer (QMS) under development at the NASA Goddard Space Flight Center (GSFC) for the 2009 Mars Science Laboratory (MSL) mission provide the necessary performance for many targets to make these measurements (refer to Table 2). However, future cost-capped New Frontiers- and Discovery/Scout-class missions require the development of more resource efficient solutions that still maintain SAM QMS-level performance. For example, Mars Scout balloon and airplane mission concepts have been developed in detail and proposed to NASA and the high science return from these platforms is widely recognized. Nevertheless, the scientific return from these aerial platforms could be

*Todd.T.King@nasa.gov; phone 301.286.4197, fax 301.286.1646 
substantially enhanced with addition of another instrument by a reduction in the mass and power of the mass spectrometers baselined for these missions. And though other space flight mass spectrometer programs such as the Strophio instrument being developed for the European Space Agency's (ESA) 2013 BepiColumbo mission to Mercury have design goals minimizing instrument mass, volume and power consumption, they do not exhibit the necessary performance, particularly in mass range and resolution for most astrobiological applications (refer to Table 2). For example, the ability to identify heavier hydrocarbons such as benzene $\left(\mathrm{C}_{6} \mathrm{H}_{6}, 78.1121 \mathrm{Da}\right)$ and standard amino acids such as alanine $\left(\mathrm{C}_{3} \mathrm{H}_{7} \mathrm{NO}_{2}, 89.1 \mathrm{Da}\right)$ and lysine $\left(\mathrm{C}_{6} \mathrm{H}_{14} \mathrm{~N}_{2} \mathrm{O}_{2}, 146.188 \mathrm{Da}\right)$ would significantly enhance astrobiology mission capability. Additionally, there are several astrobiologically significant chemical species pairs that are only slightly different in mass and require high mass resolution $(\mathrm{m} / \Delta \mathrm{m})$ for unique identification. For example, distinguishing helium-3, $\left({ }^{3} \mathrm{He}, 3.0160 \mathrm{Da}\right)$ from hydrogen deuteride $(\mathrm{HD}, 3.0219 \mathrm{Da})$ requires a mass resolution, $\mathrm{m} / \Delta \mathrm{m}>511$. In order to mass separate formaldehyde $\left(\mathrm{H}_{2} \mathrm{CO}, 30.0106 \mathrm{Da}\right)$ and ethane $\left(\mathrm{C}_{2} \mathrm{H}_{6}, 30.0470 \mathrm{Da}\right)$, even higher mass resolution of $\mathrm{m} / \Delta \mathrm{m}>824$ is needed.

Table 1: Targeted science objectives, instrument requirements, and applicable NASA missions

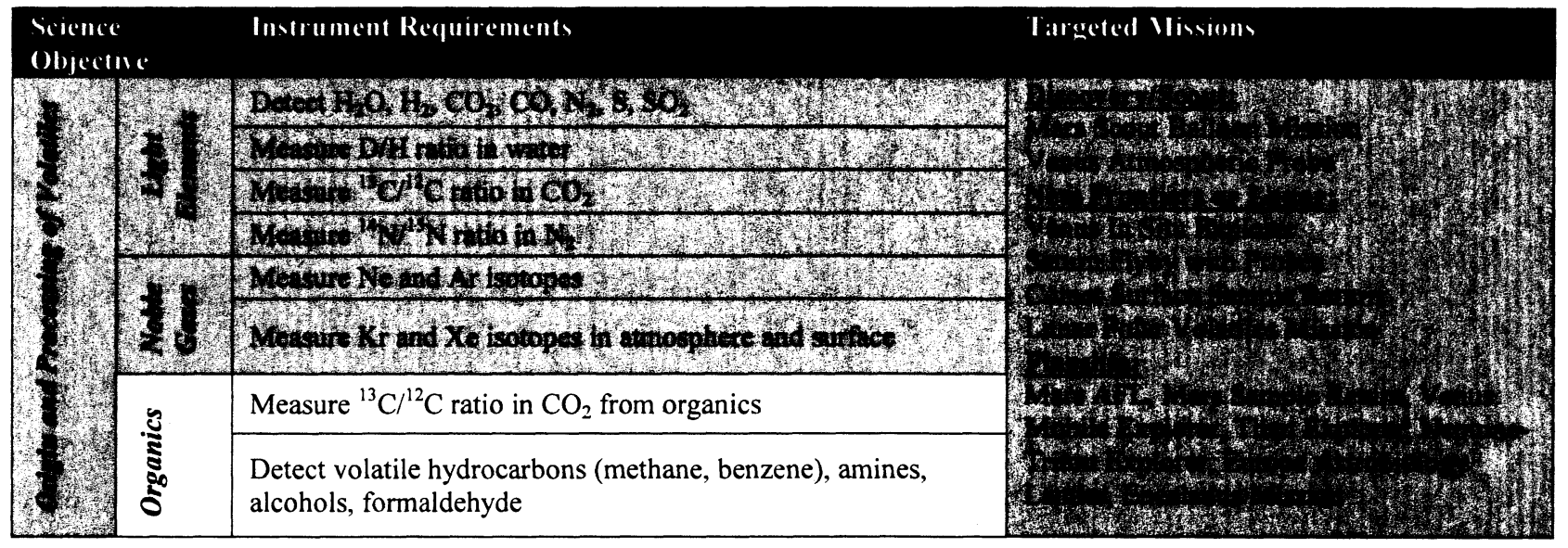

Table 2: State-of-the-art space flight mass spectrometers and the design goals of the Mini TOF-MS effort.

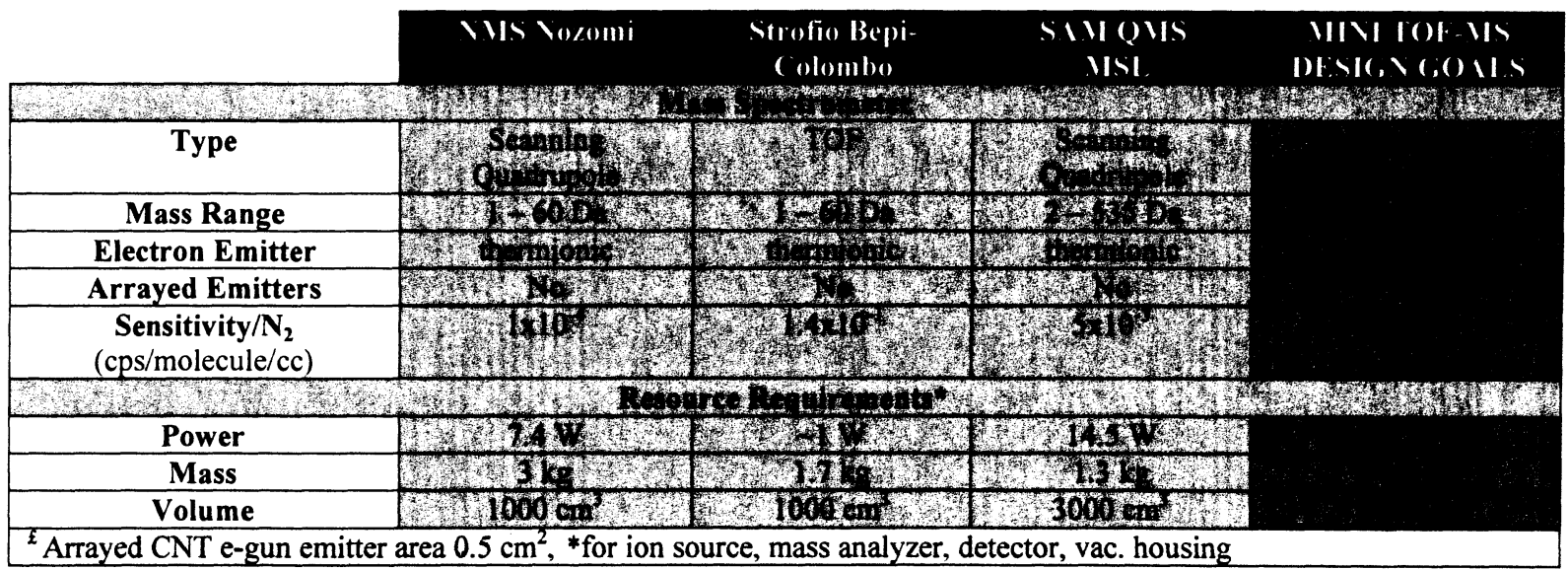

Our efforts at the NASA Goddard Space Flight Center are aimed at taking a significant step towards a substantially more resource efficient high performance mass spectrometer for space use. Our technical approach to instrument development emphasizes a simple, miniature reflectron time-of-flight mass spectrometer (Mini TOF-MS) architecture featuring an extended low power ion source that delivers the high performance required by the mission science objectives identified above. Figure 1 illustrates examples of the mission science achievable with the Mini TOF-MS as well as a relative scale 
comparison to the state-of-the-art SAM QMS. We have selected a time-of-flight mass separation mechanism over competing technologies, e.g. quadrupole or ion trap, because of the high attainable mass range, simplicity of design, fast integration time, and low power consumption that are characteristic of TOF-MS systems. We employ a combination of tactics to achieve high mass resolution that include minimizing the spatial distribution of the ion packet by designing gridless, low voltage, micron-scale ion optics with a reduced ionization volume along the ion flight direction. A gridless non-linear reflectron compensates for initial thermal kinetic energy spread, and our TOF-MS will achieve high sensitivity $\left(\sim 10^{-3} \mathrm{cps}_{\text {part }} \mathrm{cm}^{-3}\right)$ by densely arraying CNT e-gun field emitters to extend the ionization volume in the ion source. Our design goals for the Mini TOF-MS development are listed in Table 2.

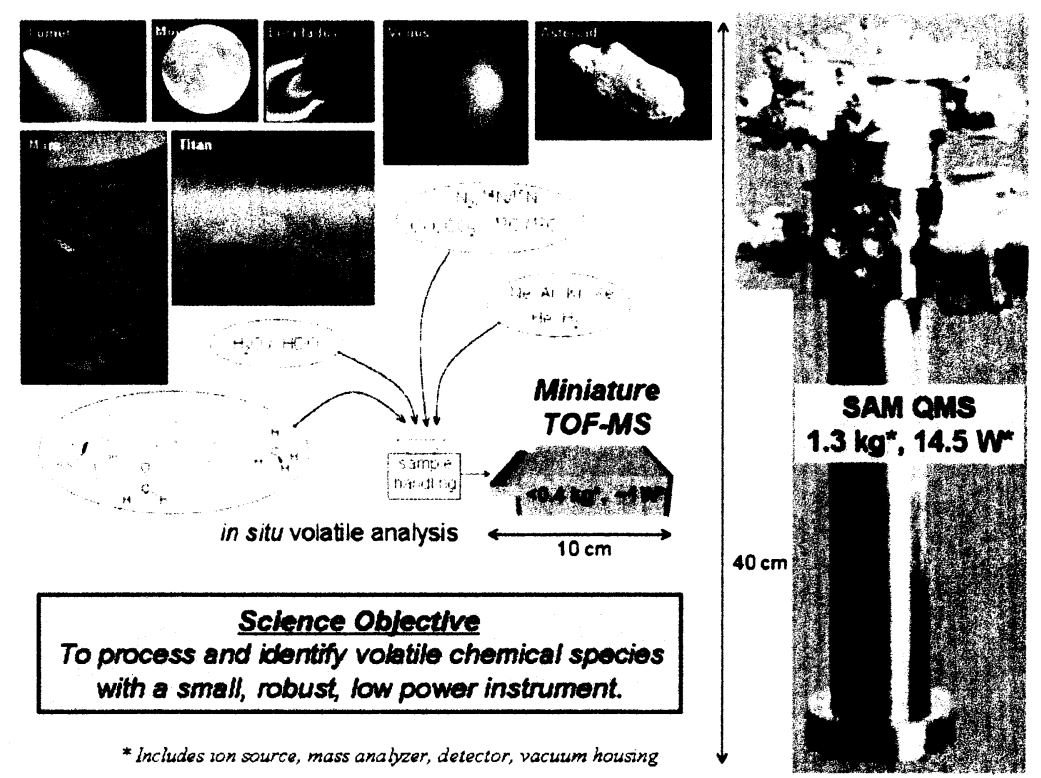

Fig. 1. The Mini TOF-MS will address a range of science objectives by enabling in situ chemical and isotopic measurements of volatile species to constrain the origin and processing of light elements $(\mathrm{C}, \mathrm{H}, \mathrm{O}, \mathrm{N}$, and $\mathrm{S})$, the noble gases, and prebiotic organic compounds within a small instrument package as compared to SAM QMS.

We make extensive use of electrodynamic computer simulations to guide the Mini TOF-MS design process. In a TOFMS system employing electron impact ionization, instrument performance is dependent upon the flight of charged particles: the flight of individual electrons comprising the electron beam defines the ionization volume within the ion source and the flight characteristics of positive ions accelerated through the mass analyzer to the detector ultimately dictate the resolution and sensitivity. Electrodynamic simulations enable an efficient iterative process of modeling different electrode geometries and defining optimal electrode voltage levels, characterizing electron and ion optic performance, and identifying subsequent design improvements. The ability to simulate and thoroughly vet the end-toend performance of the Mini TOF-MS design in terms of resolution and sensitivity prior to any expensive hardware builds has been a valuable asset during this technology development effort.

\section{DESIGN CONCEPT}

Our Mini TOF-MS concept follows basic reflectron TOF-MS in design and operation and is illustrated in the schematic cross section shown in Figure 2. In this reflectron TOF-MS arrangement, a volatile species is introduced to the ion source through a gas inlet and is ionized by an electron beam (red dotted arrow) via electron impact. Ion optics apply a voltage pulse to accelerate all the ions (blue dashed arrow) to a constant energy toward the reflectron mass analyzer. Mass separation occurs within the reflectron mass analyzer because ion velocities are inversely proportional to the square root of their masses such that lighter ions travel faster than heavier ions. The reflectron also compensates for the initial kinetic energy spread of the ions by applying a retarding potential that reverses the direction of ion motion which reproduces the ion space focus plane at the reflected $\mathrm{MCP}$ detector, effectively extending the flight path length by a factor of two over linear mode TOF-MS operation. Each ion impact is registered by the microchannel plate (MCP) detector and the TOF is recorded by an application specific integrated circuit (ASIC) electronics board. The 
accumulated TOF data is then processed to generate a mass spectrum. A more thorough description of TOF-MS operation can be found elsewhere ${ }^{3}$.

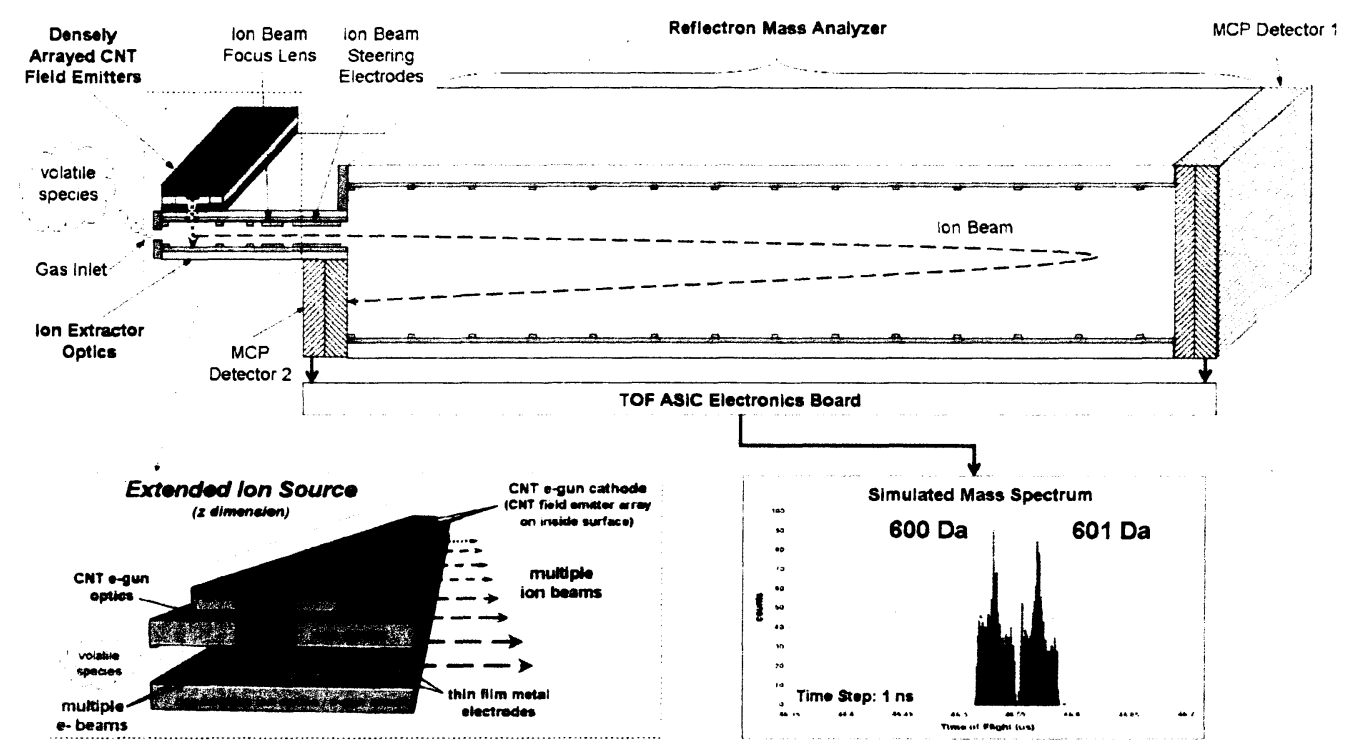

Fig. 2. Schematic cross section of the Mini TOF-MS design concept illustrating the constituent components: densely arrayed carbon nanotube field emitter array, controllably doped resistive glass ion optics and reflectron mass analyzer, microchannel plate detectors, TOF ASIC electronics. Scaleable parallel-plate geometry allows ion source extension for maximum sensitivity.

Our approach towards miniaturization of this basic reflectron TOF-MS architecture is to apply nano- and microfabrication techniques to develop core high performance, low power mass spectrometer components. For example, we utilize monolithic, low voltage, $(<800 \mathrm{~V})$ ion optics and a reflectron mass analyzer that are configured in a parallel-plate geometry from controllably-doped resistive glass substrates ${ }^{\dagger}$. Thin film metal traces are patterned and deposited onto adjacent ion optic or reflectron substrates to form pairs of gridless electrodes that generate the precise electric fields required to accelerate and guide charged particles through the Mini TOF-MS system without the sensitivity loss and contamination issues associated with grid electrodes. Use of resistively-doped substrates as the primary structural members of the ion optics and reflectron also eliminates the need for extraneous stray electric field shielding in those regions. Secondly, we take advantage of the parallel-plate geometry to create an extended ion source that features densely-arrayed, low power carbon nanotube field emitters (CNT e-gun) as the electron sources. The scalable aspect of the ion source provides multiple ionization sites for increased sensitivity without compromising resolution. Finally, the high degree of microfabrication compatibility shared by the CNT e-gun and ion optics both in terms of processing techniques and common materials (silicon and silicon dioxide substrates, metal thin films) allows straightforward integration of the CNT e-gun with the ion optic substrate to produce a robust, small volume ion source.

\section{SIMULATION METHODOLOGY AND PERFORMANCE CHARACTERIZATION}

An electrodynamics simulation software package, SIMION 3D Ion and Electron Optics Simulator (v8.0.4) $)^{\ddagger}$, was used to guide the design of the Mini TOF-MS concept by establishing three-dimensional electrostatic potential array geometries, calculating the electric field of the free space between potential arrays, simulating the flight trajectories of electrons and ions created within the model, and characterizing the end-to-end instrument performance in terms of mass resolution and sensitivity. The potential arrays define the geometry for all solid electrode structures in the design. Insulative structures are not explicitly included in the model and are treated as free space. Potentially detrimental charging effects caused by insulative surfaces are minimized at the design level: all insulative surfaces in the Mini TOF-MS design are either

\footnotetext{
${ }^{+}$FieldMaster ${ }^{\mathrm{TM}}$ Ion Guides / Drift Tubes, Photonis USA, Inc., www.photonis.com.

‡ SIMION 3D Ion and Electron Optics Simulator (v8.0.4), Scientific Instruments Services, Inc., www.simion.com.
} 
- shielded from direct charged particle beam impingement by conductive/resistive structures or electrostatically blocked by maintaining a $4: 1$ ratio between electrode length and electrode gap where an insulative surface is exposed ${ }^{4}$. The potential array geometries were for the CNT e-gun, ion optics, reflectron mass analyzer, and MCP detector were first modeled and tested as individual component instances and then later combined in an ion optics workbench to form the integrated model of the Mini TOF-MS device. The interfaces between components share a common potential.

The electric potential throughout the entire model was determined by solving the Laplace equation via an over-relaxation finite difference technique at every non-electrode point subject to the boundary conditions imposed by the defined electrodes. The electric field was then computed by taking the gradient of the electric potential. A full description of the computation methods employed by the SIMION software is described SIMION Version 8.0 User Manual (Appendix $\mathrm{H}$ ). A fine grid spacing was defined for each modeled component to ensure the accuracy of the calculated electric field. The grid spacing ranged between $0.050 \mathrm{~mm}$ and $0.5 \mathrm{~mm}$ per grid unit depending on the level of structural detail.

In order to characterize the charged particle optic performance of the CNT e-gun, ion optics and reflectron mass analyzer, 60,000 charged particles (electrons in the case of the CNT e-gun and positive ions in the case of the ion optics and reflectron) were randomly generated and allowed to move within the model as dictated by the calculated electric field. The properties of the electrons and ions were allowed to randomly vary either between user-specified limits or following known distributions (e.g. Maxwell-Boltzmann distribution describing the kinetic energy of gas or Lorentz distribution describing spectral peak shape due to collision broadening) to most accurately represent laboratory conditions. The properties that defined a charged particle included the particle charge, mass, birth coordinates, initial trajectories direction, and initial average energy.

After specifying the operating voltages for all component potential arrays of the integrated Mini TOF-MS model, the end-to-end performance was characterized by simulating the flight of the randomly generated electrons and ions through the calculated electric field of the model to determine the instrument resolution and sensitivity.

The instrument resolution was determined by restricting the ion masses to two values separated by $1 \mathrm{Da}$. Sixty thousand such ions in equal proportions were randomly generated within the ionization volume (as defined by the boundaries of the ionizing electron beam within the ion source) and accelerated instantaneously (i.e. zero rise time voltage pulse) into the reflectron mass analyzer and then to the MCP detector 2. The TOF data of all ions arriving at the detector was recorded and binned into time steps consistent with response time of TOF ASIC electronics, and plotted into a TOF histogram. The two ion masses were adjusted until a histogram characterized by two TOF spectral peaks as defined by a $90 \%$ peak-valley criterion was produced. The TOF spectral resolution and the instrument mass resolution are related and defined as

$$
\frac{m}{\Delta m}=\frac{t}{2 \Delta t}
$$

where $m$ is the mass of one of the ion species, $\Delta m$ is the mass difference between the two ion species, $t$ is the ion TOF, and $\Delta t$ is the TOF difference between the two ion species.

The instrument sensitivity was calculated by first considering a cylindrical ionization volume defined by the ionizing electron beam dimensions as shown in Figure 3. $I_{e}$. is the current emitted from the electron impact ionization source, $A$

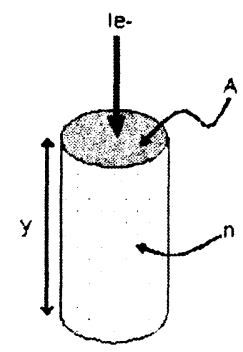

Fig. 3: Schematic representation of idealized ionization volume bounded by the dimensions of an electron beam.

is the cross sectional area of the ionization volume perpendicular to the electron beam, $y$ is the height of the ionization volume, and $n$ is the number of molecules inside the ionization volume. The ionization efficiency, $E_{i}$, is the ratio of the 
total cross sectional area of all $n$ molecules over the total cross sectional area of the ionization volume and is expressed as

$$
E_{i}=\frac{n \sigma_{i}}{A},
$$

where $\sigma_{l}$ is the electron impact cross section for ionization for molecular species $i$. Equation (2) can be re-expressed in terms of molecular density, $d$, by normalizing by the ionization volume,

$$
E_{l}=y d \sigma_{i} .
$$

The ionization efficiency can also be evaluated in terms of the ratio of rate of ionized charges generated, $I_{l}$, over the total rate of electron charges available for ionization, $I_{e^{-}}$,

$$
E_{i}=\frac{\left(I_{i} / e\right)}{\left(I_{e-} / e\right)},
$$

where $e$ is the absolute value of the elementary charge carried by a singly charged ion or electron. Equating (3) and (4) and rearranging yields the total number of ions created by the electron beam,

$$
\left(\frac{I_{i}}{e}\right)=\left(\frac{I_{e-}}{e}\right) y d \sigma_{i} .
$$

In any mass spectrometer the probability that an ion successfully arrives at the detector is determined by the transmission efficiency, $E_{l}$, which is dependent upon characteristics of the ion source and intervening ion optics. Thus the rate of ion arrival at the detector, $I_{D e l}$, can be expressed as

$$
\left(\frac{I_{D e t}}{e}\right)=\left(\frac{I_{i}}{e}\right) E_{t}=\left(\frac{I_{e-}}{e}\right) y d \sigma_{i} E_{t} .
$$

Finally the sensitivity of the mass spectrometer, $S_{i}$, to a molecular species $i$ can be expressed by normalizing (6) by the molecular density such that

$$
S_{i}=\left(\frac{I_{e-}}{e}\right) y \sigma_{i} E_{t} .
$$

The transmission efficiency, $E_{t}$, can be determined by tracking the ion trajectories in the simulated model and recording the statistics of ion arrival at the detector. The ionization volume height, $y$, is defined by the modeled gap between the ion optics across which the electron beam passes. The rate of electron charge or electron beam current, $I_{e-\text {, }}$ is measured through laboratory experimentation and the ionization cross section, $\sigma_{i}$, for specific molecular species can be found in the literature.

Based upon the simulated performance (mass resolution and sensitivity), Mini TOF-MS design improvements were identified and implemented in subsequent design revisions. This design process was iterated until the desired prototype design criteria were achieved.

\section{COMPARISON BETWEEN SIMULATED AND LABORATORY PERFORMANCE}

Confirmation of the predictive accuracy of these simulation techniques was obtained by comparing the mass spectra obtained by an early version of a miniature TOF mass spectrometer of a different design produced at NASA GSFC with SIMION computer models and simulated performance. The primary goal of this early version was to demonstrate the efficacy of microfabrication technology in producing a miniaturized mass spectrometer. Discrete electrodes were individually fabricated out of silicon and hand-assembled to form an ion optical component. A traditional thermionic emitter was incorporated to provide electron impact ionization within the ion source. The ion source was combined with 


$$
\because
$$

$\because \quad$ a resistive glass field-free drift tubet and a MCP detector $\$$ to form a simple mass spectrometer configured for lower resolution, linear TOF operation within a small volume package $\left(<100 \mathrm{~cm}^{3}\right)$. The core elements of our first miniature mass spectrometer prototype are shown fully integrated in the photograph in Figure 4.

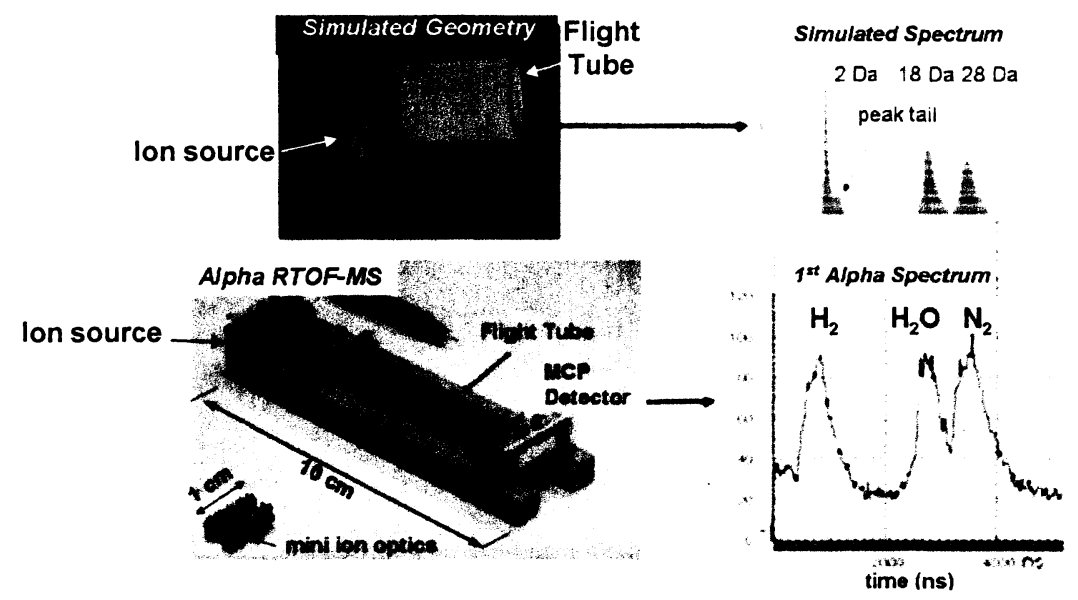

Fig. 4: An early miniature TOF mass spectrometer utilizing MEMS components and low resolution TOF spectrum. Electrodynamic modeling of the as-built hardware generated the simulated TOF spectrum. The excellent agreement between the simulated and actual spectra demonstrates the accuracy of these predictive methods.

This mass spectrometer core was installed within a high vacuum chamber and connected via electrical feedthroughs to laboratory-scale voltage pulse control electronics, TOF electronics, and a data acquisition system. Performance testing of this early prototype was conducted at a pressure of $10^{-6}$ torr and yielded the low resolution TOF spectrum shown in Figure 4. The spectrum was characterized by three broad peaks associated with masses $2 \mathrm{Da}\left(\mathrm{H}_{2}\right), 18 \mathrm{Da}\left(\mathrm{H}_{2} \mathrm{O}\right)$, and 28 $\mathrm{Da}\left(\mathrm{N}_{2}\right)$ respectively, and was in excellent agreement with simultaneous results from a residual gas analyzer (RGA) ${ }^{* *}$ sampling the vacuum chamber volume.

All as-built conductive surfaces of this early mass spectrometer prototype were modeled as electrodes within a potential array as shown in Figure 4 and the electrical operation of the modeled device in linear mode was simulated using identical control voltage parameters employed during laboratory testing. Equal proportions of the masses identified by the RGA results $(2 \mathrm{Da}, 18 \mathrm{Da}$, and $28 \mathrm{Da}$ ) were randomly generated within the ionization volume of the model's ion source and accelerated towards the detector plane. The TOF of each ion impact at the detector plane was recorded and a simulated TOF spectrum was generated and is shown in Figure 4. Comparison with the laboratory results shows that the simulated spectrum accurately predicts the TOF peak positions. In addition, the simulated TOF spectrum also accurately predicts the peak shape which is characterized by a peak broadening towards higher TOF. The peak broadening toward higher TOF in the simulated spectrum was induced by continued ion production by the electron beam throughout the long (312 ns) voltage pulse out phase of ion acceleration. The agreement between the simulated and laboratory-obtained TOF spectra confirms the accuracy of these simulation methods and their efficacy for guiding TOF-MS instrument design and characterizing performance.

\section{MINI TOF-MS DESIGN SIMULATION AND CHARACTERIZATION}

In order to realize the Mini TOF-MS design, a proof-of-concept laboratory prototype was modeled and its performance simulated. The design goals of this proof-of-concept laboratory prototype were to demonstrate high mass resolution ( $>$ $500)$ with sufficient sensitivity $\left(>1 \times 10^{-5} \mathrm{cps} /\right.$ molecule $\left.\mathrm{N}_{2} / \mathrm{cc}\right)$ within a small modeled volume $\left(<1000 \mathrm{~cm}^{3}\right)$ while accomodating instrument packaging constraints to simplify future hardware integration. Additional design emphasis was placed upon reducing overall development cost such that in-hand parts and existing components were incorporated into the proof-of-concept prototype model and simulation. The resulting proof-of-concept Mini TOF-MS prototype alters the design discussed in Section 2 in the following ways: 1) a stand-alone CNT e-gun is employed rather than one

$\$ 8 \mathrm{~mm}$ Miniature TOF Detector, Photonis USA Inc., www.photonis.com.

${ }^{* *}$ RGA300, Stanford Research Systems, www.thinksrs.com. 
directly deposited to the ion optics to facilitate independent component-level CNT e-gun testing prior to integration, 2) the ion source is not extended for maximum sensitivity to enable use of existing, pre-sized resistive glass ion optic substrates, 3) sidewall electrode plates are included on the ion optics and reflectron mass analyzer to provide stray electric field shielding, and 4) an existing $8 \mathrm{~mm}$ miniature TOF detector ${ }^{\$}$ is utilized. A modeled prototype based upon these design requirements will be sufficient to prove the Mini TOF-MS concept through simulated performance. Subsequent prototypes can be optimized further to demonstrate higher sensitivity (extended ion source and custom MCP detector) and additional packaging robustness (i.e. a fully integrated CNT e-gun), as resources permit.

\subsection{Ion Source Model}

The modeled electrode geometry of the proof-of-concept Mini TOF-MS ion source is comprised of a CNT e-gun and resistive glass ion optics and is shown in cross section in Figure 5a (inset illustrates the same geometry from an isometric
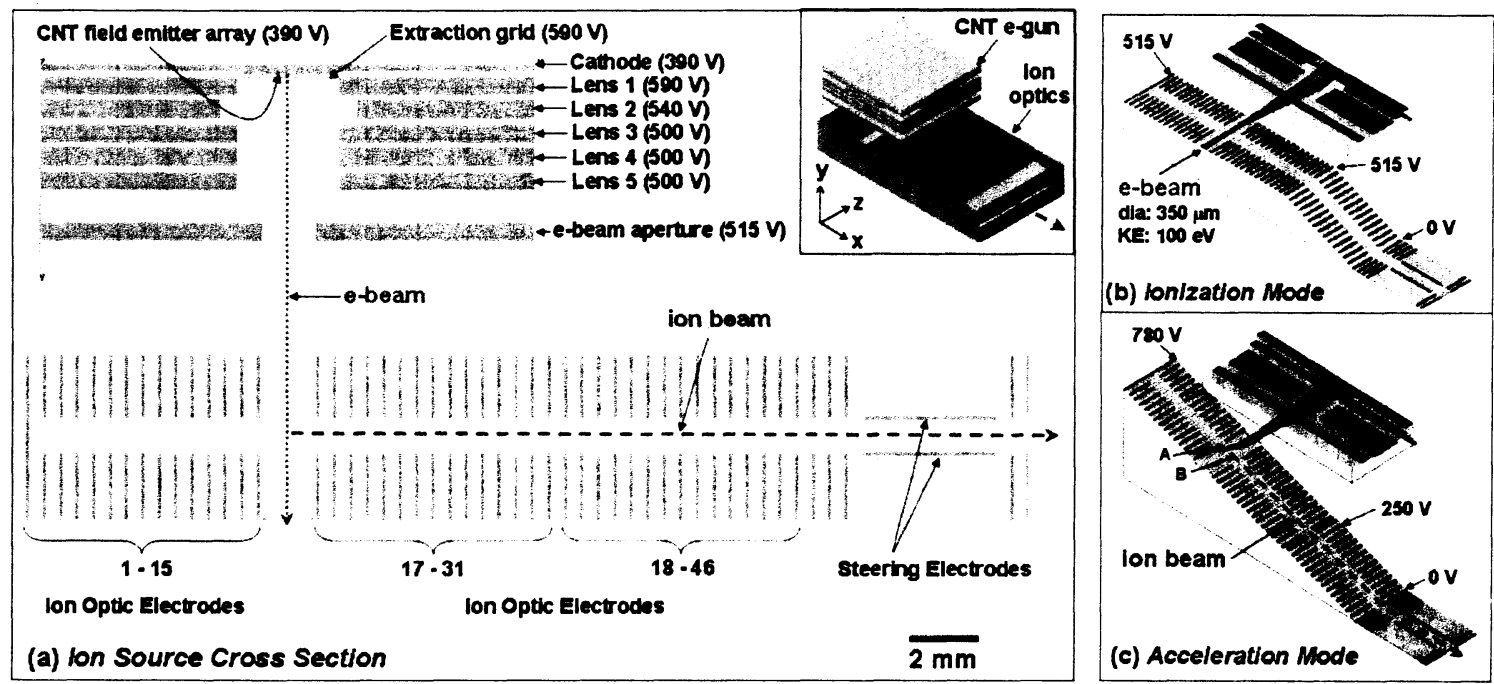

Fig. 5: (a) Cross section of modeled ion source electrode geometry with isometric view in inset. Continuous resistive glass substrates of ion optics modeled as series of discrete aperture electrodes. Potential energy plots of ion source cross section illustrating ion source operational modes: (b) ionization mode and (c) acceleration mode.

perspective). The CNT e-gun electrode geometry consisted of a CNT cathode substrate, an extraction grid (modeled as an ideal grid), and five electrostatic lenses. To simulate CNT e-gun operation, tens of thousands of electrons were randomly generated within a initial kinetic energy range of $0.025 \mathrm{eV}-0.027 \mathrm{eV}$, with initial trajectories randomized about $360^{\circ}$ in elevation and azimuth angle, and within a plane defined by the CNT tips of a virtual $2 \times 2 \mathrm{~mm}$ CNT field emitter array deposited and grown to a height of approximately $10 \mu \mathrm{m}$ directly onto the cathode substrate. A bias voltage $(200 \mathrm{~V})$ was placed upon the extraction grid to accelerate the electrons while electrostatic lenses focused the electron beam and attenuated the electron kinetic energy within the ion optic gap $(y=1 \mathrm{~mm})$ to $70 \mathrm{eV}-100 \mathrm{eV}$ in order to maximize ionization efficiencys. The extraction potential was selected based upon prior laboratory tests of earlier . CNT e-gun prototypes. The potentials assigned to the electro-optic lenses, listed in Figure 5a, resulted in an average electron beam diameter of $350 \mu \mathrm{m}$ and a final electron kinetic energy of $100 \mathrm{eV}$ within the ion optic gap as illustrated in Figure $5 \mathrm{~b}$. The ionization volume of the ion source was defined by the ion optic gap $(y=1 \mathrm{~mm})$ and the average diameter of the focused electron beam $(350 \mu \mathrm{m})$.

The continuous resistive surfaces of the monolithic glass substrates to be used in the construction of the ion optics were modeled as a series of discrete aperture electrodes also shown in Figure 5a. The aligned apertures simulated the ion optic gap $(y=1 \mathrm{~mm})$ between the top and bottom ion optic substrates. The fine aperture electrode spacing of $0.5 \mathrm{~mm}$ ensured close approximation to the electric field generated by two resistive glass substrates patterned with linear thin film electrodes and arranged in a parallel plate geometry. Two electrodes were modeled near the ion optic exit to facilitate ion beam steering in the Y dimension. The ion source was simulated to function in two operational modes: ionization mode and acceleration mode. The voltage combinations defining the two modes are illustrated in the two 
$\because \quad$ plots in Figure $5 \mathrm{~b}$ and Figure $5 \mathrm{c}$ where the vertical axis corresponds to the potential energy at every point within the modeled ion source cross section. During ionization mode, the electron beam (labeled e-beam in Figure $5 \mathrm{~b}$ ) transects the ion optic gap which in this vicinity (ion optic electrodes $1-31$ ) and is characterized by a constant $515 \mathrm{~V}$ potential energy surface (zero electric field). Ions are generated via electron impact ionization of neutral volatile species which occurs within the ionization volume defined by the electron beam. Due to the constant potential energy surface, ions are not accelerated in this mode. The voltage combination represented by the acceleration mode, defines the constant electric field generated by a voltage pulse that accelerates the ions out the ion source exit (the resultant ion beam is schematically illustrated by the blue dashed arrow). The steering electrodes are activated to deflect the ion beam towards the reflected MCP detector 2. Two pairs of voltage matched sidewall electrodes enclosed the sides of the ion optics to block stray electric fields from disrupting ion flight within the ion source (visible in inset of Figure 5a).

\subsection{Reflectron Mass Analyzer Model}

The modeled electrode geometry of the reflectron mass analyzer is shown in Figure 6a. As was the case for the ion optics, a series of discrete aperture electrodes were used to model the electric field generated by the resistive surfaces
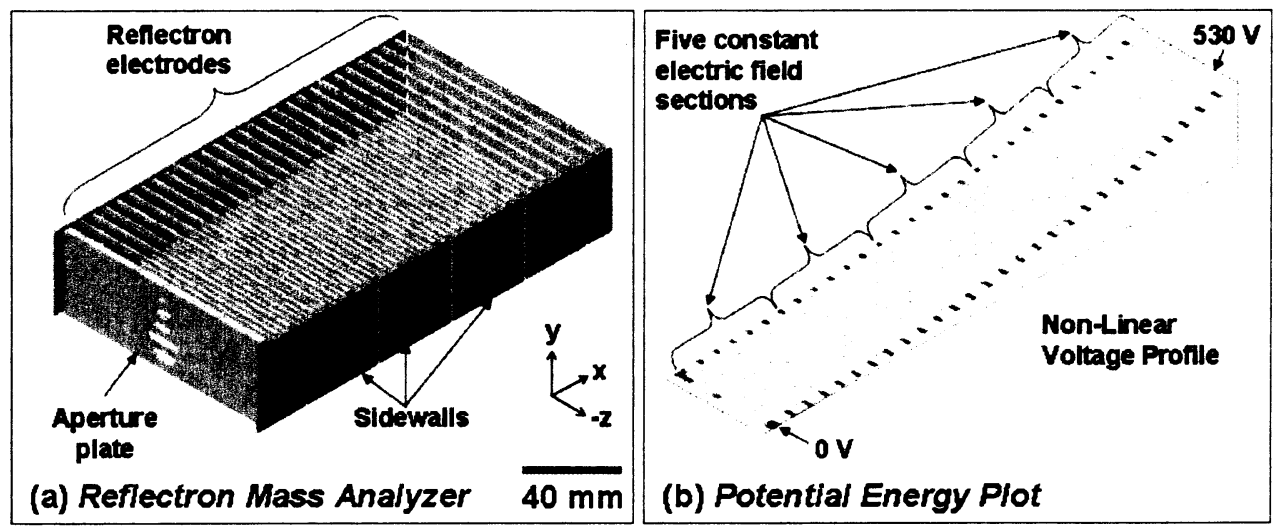

Fig. 6: (a) Isometric view of modeled reflectron mass analyzer electrode geometry. Continuous resistive glass substrates of reflectron modeled as series of discrete aperture electrodes. (b) Potential energy plot of reflectron cross section illustrating non-linear voltage profile along reflectron axis.

patterned with thin film linear electrodes arranged in a parallel plate configuration. The aligned apertures simulated the reflectron gap $(25 \mathrm{~mm})$ between the top and bottom reflectron substrates and an electrode spacing of $5 \mathrm{~mm}$ ensured a close approximation to the electric field generated by adjacent continuous resistive surfaces defined by linear thin film electrodes. The voltage gradient along the length of the reflectron mass analyzer employs five constant electric field sections to approximate a non-linear voltage profile of an ion mirror with high order kinetic energy compensation ${ }^{6}$ and is illustrated in the potential energy plot of Figure $6 \mathrm{~b}$. Besides enhancing mass resolution with $5^{\text {th }}$ order kinetic energy compensation, the non-linear reflectron voltage gradient also facilitates the gridless electrode design: the zero electric fields at the entrance/exit of the reflectron do not introduce a sharp discontinuity in the electric field which can divert ion trajectories leading to lower sensitivity. Six pairs of voltage matched sidewall electrodes enclosed the sides to block stray electric fields from disrupting ion flight within the reflectron mass analyzer. Though the reflectron width $(80 \mathrm{~mm})$ is oversized for this proof-of-concept prototype, it was designed this way to facilitate investigations into sensitivity enhancements involving an extended ion source. The length $(140 \mathrm{~mm})$ and width $(80 \mathrm{~mm})$ of the reflectron were set by the maximum resistive glass substrate dimensions commercially available $\dagger$.

\subsection{Ion Space Focus Plane}

The modeled ion source and reflectron mass analyzer components were first combined into a virtual ion optic workbench and characterized in SIMION in order to locate the reflected ion space focus plane and thus determine the optimal position for detector placement. Since randomly generated iso-mass ions may be birthed anywhere within the finite ionization volume (i.e. $350 \mu \mathrm{m}$ electron beam diameter), upon initiation of acceleration mode ions that were formed closer to back of the ion source (point $\mathrm{A}$ in Figure 5c) will attain a slightly higher velocity than ions that were formed closer to the front of the ion source (point B in Figure 5c) due to the difference in potential energy at the two locations. 
At some point after the ion source, the faster ions will catch up with the slower ions. This location is referred to as the space focus plane and represents the position of sharpest temporal focus of the ion packet in a TOF-MS system. The addition of a reflectron reproduces this temporal focus at a new location known as the reflected space focus plane. Maximum instrument mass resolution is attained by positioning the detector at the reflected space focus plane.

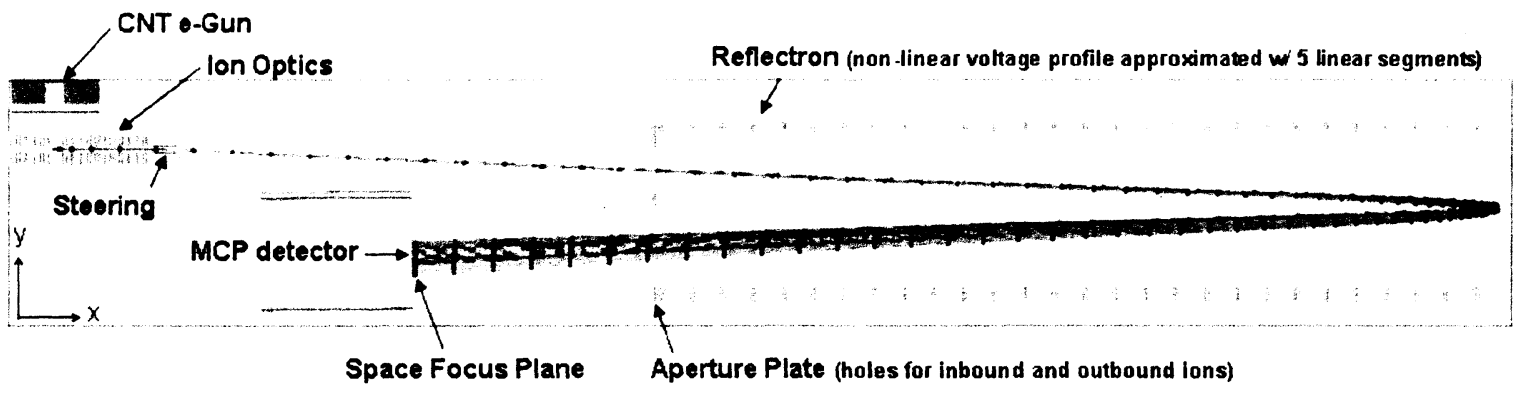

Fig. 7: Cross section of ion optic workbench with ideal iso-mass ion beam trajectories with higher (green) and lower (blue) initial kinetic energies. Red dots denote time markers at $0.5 \mu$ intervals. Coincidence of time markers identifies unique reflected space focus plane location.

The location of the reflected space focus plane was simulated from the combined ion source and reflectron by birthing 10 ideal ions (zero initial kinetic energy and user-specified birth coordinates) at the extreme leading (point B) and trailing (point $\mathrm{A}$ ) edges of the ionization volume, accelerating them into the reflectron mass analyzer in acceleration mode, and tracking their trajectories with time markers set at $0.5 \mu$ s intervals as shown in the cross sectional view of Figure 7. The trajectories of the faster ions from point A and of the slower ions from point $B$ are traced in green and blue, respectively. The simulated results reveal a coincidence of ion time markers at a unique location within the model identifying the reflected space focus plane and the optimal detector position.

\subsection{Integrated Mini TOF-MS Model and Mass Resolution}

The modeled ion source and reflectron mass analyzer components were combined with a modeled $8 \mathrm{~mm}$ miniature TOF detector oriented to receive ions at the reflected space focus plane to form an integrated proof-of-concept Mini TOF-MS prototype shown in Figure 8a. The spaces between adjacent components (instances in SIMION terminology) were characterized by a constant $515 \mathrm{~V}$ between the CNT e-gun and the ion optics and common ground potential between all other components to ensure realistic ion trajectory simulations that were subjected to all non-zero electric fields. Subsequent hardware builds based upon this design geometry will maintain these inter-component zero electric fields. The component positions were optimized to find the configuration that offered the best overall combination of instrument mass resolution and sensitivity and packaging simplicity. The final modeled volume of the Mini TOF-MS

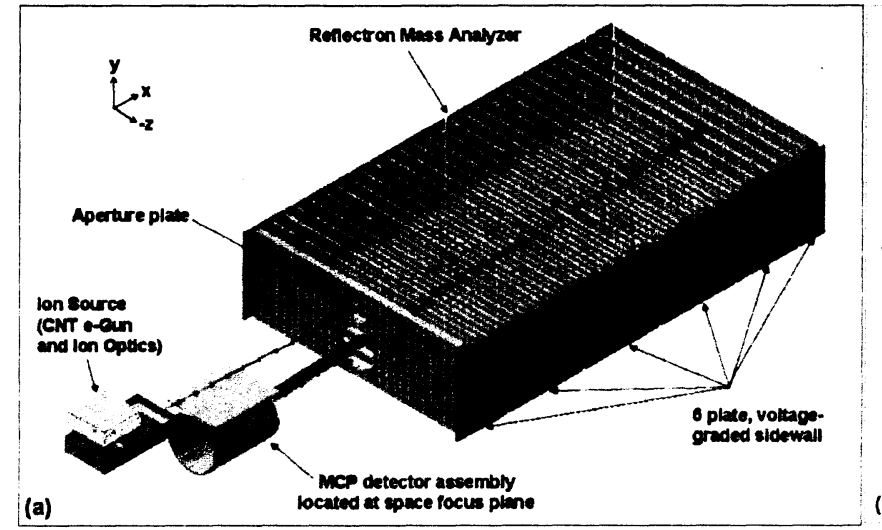

Fig. 8: (a) Isometric view of modeled integrated Mini TOF-MS prototype. resolution at $600 \mathrm{Da}$.

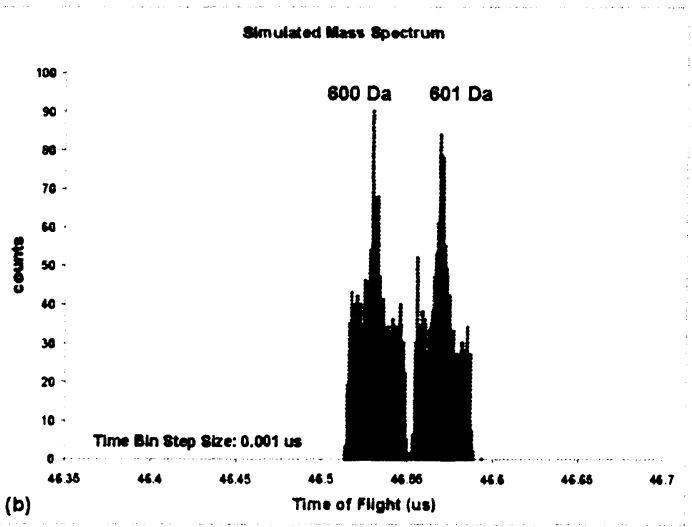

(b) Simulated mass spectrum indicating unit mass 
?

$\because$ prototype was $<840 \mathrm{~cm}^{3}$.

The mass resolution of the modeled Mini TOF-MS prototype was determined following the procedure identified in Section 3. Assuming $1 \mathrm{~ns}$ time step binning intervals, the resultant TOF spectrum is shown in Figure 8b. Application of (1) on the two distinct TOF peaks characterizing this TOF spectrum demonstrates that the modeled Mini TOF-MS prototype design achieves a mass resolution, $m / \Delta m$, of $>600$, satisfying our stated design goal of $m / \Delta m>500$ for proofof-concept. The simulated Mini TOF-MS prototype mass resolution also surpasses that of the current state-of-the-art SAM QMS (unit resolution at $535 \mathrm{Da}$ ) and is sufficient to uniquely identify astrobiologically important chemical pairs such as helium-3, $\left({ }^{3} \mathrm{He}, 3.0160 \mathrm{Da}\right)$ and hydrogen deuteride (HD, 3.0219 Da) for approximately a third of the instrument volume.

\subsection{Mini TOF -MS Sensitivity}

The sensitivity of the modeled Mini TOF prototype was calculated following the procedure described in Section 3 . In spite of the undersized sensor area of the modeled detector $(8 \mathrm{~mm}$ diameter $)$, statistical analysis of the simulated trajectories of all 60,000 randomly generated ions indicate a Mini TOF-MS prototype transmission efficiency, $E_{l}$, of $6 \%$. Prior laboratory experimentation on early CNT e-gun prototypes have demonstrated electron emission currents, $I_{e-\text {, }}$ on the order of $1 \times 10^{-5} \mathrm{~A}$ at the extraction grid $^{+\dagger}$. Assuming an electron impact cross section for ionization, $\sigma_{i}$, corresponding to diatomic nitrogen ${ }^{7}\left(\mathrm{~N}_{2}\right)$ of $2.5 \times 10^{-16} \mathrm{~cm}^{2}$ for incident electron energies of $100 \mathrm{eV}$ as determined in the model, application of Equation (7) yields a calculated Mini TOF-MS prototype sensitivity of $9 \times 10^{-5} \mathrm{cps} / \mathrm{molecule}$ $\mathrm{N}_{2}$ /cc. Though this level of sensitivity is sufficient to prove out the Mini TOF-MS concept, the modeled prototype could be readily improved by replacing the undersized $8 \mathrm{~mm}$ diameter TOF detector with a larger, custom-shaped MCP detector. Simulations of this slightly modified configuration demonstrate a higher transmission efficiency of $15 \%$ which results in a higher instrument sensitivity of $2 \times 10^{-4} \mathrm{cps} /$ molecule $\mathrm{N}_{2} / \mathrm{cc}$ for a single ion source.

Further improvements in sensitivity can be achieved by taking advantage of the scaleable parallel plate geometry of the Mini TOF-MS design through extension of the ion source as a way to increase the ionization volume as depicted in Figure 2. By considering ion source extension as multiples of a single ion source, then an ion source multiplicative factor, $M$, can be incorporated into (7) such that the overall instrument sensitivity is described as

$$
S_{i}=\left(\frac{I_{e-}}{e}\right) y \sigma_{i} E_{t} M
$$

Taking the slightly modified Mini TOF-MS configuration with a larger MCP detector as the as the basis for the sensitivity of a single ionization volume achievable with a $2 \times 2 \mathrm{~mm} \mathrm{CNT}$ field emitter array, we can estimate the sensitivity of a multiple ionization device. Extension of the ion source by a multiplicative factor of 25 results in a 50 $\mathrm{mm}$ wide ion source that fits easily within the maximum resistive substrate width $(80 \mathrm{~mm})$ while leaving sufficient space $(15 \mathrm{~mm})$ to avoid potential fringing field effects along the instrument edges. Application of (8) then provides a sensitivity estimate for a Mini TOF-MS with an extended ion source of $6 \times 10^{-3} \mathrm{cps} / \mathrm{molecule} \mathrm{N}_{2} / \mathrm{cc}$, a value on par with current-state-of-the-art mass spectrometers such as the SAM QMS. Preliminary simulation efforts suggest that sensitivity enhancement due to ion source extension is possible as long as the electric field uniformity with respect to the instrument width ( $\mathrm{Z}$ dimension) is carefully maintained.

\subsection{Mini TOF -MS Power Requirements}

Based upon the specified design geometry, simulated electrical operating conditions and instrument performance results above, the overall power required to operate the proof-of-concept Mini TOF-MS prototype (non-extended ion source, 8

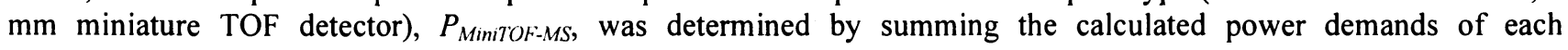
component,

$$
P_{\text {MinTOF }-M S}=P_{C N T}+P_{I O}+P_{\mathrm{Re} f}+P_{\text {Det }},
$$

where $P_{C N T}, P_{I O}, P_{R e f}$, and $P_{D e t}$ are the power consumed by the CNT e-gun, ion optics, reflectron mass analyzer, and MCP detector, respectively.

\footnotetext{
${ }^{++}$With proper alignment in subsequent prototypes, it is expected that the same emission current is possible at the anode.
} 
The power required to operate the non-extended $\left(2 \mathrm{~mm} \times 2 \mathrm{~mm} \mathrm{CNT}\right.$ field emitter array) CNT e-gun, $P_{C N T}$, can be $\cdots$ described by Joules's Law,

$$
P_{C N T}=I_{e-} V_{\text {extract }},
$$

where $V_{\text {extract }}$ is the bias voltage required to extract the emission current. Laboratory experiments on early CNT e-gun prototypes have demonstrated electron emission currents, $I_{e^{-}}$, of $1 \times 10^{-5} \mathrm{~A}$ with an applied extraction voltage of $200 \mathrm{~V}$. Thus from (10), $P_{C N T}$ is $2 \times 10^{-3} \mathrm{~W}$.

The electrical power consumed by the ion optics, $P_{I O}$, consists of two elements: the power required to establish the static voltage gradients in ionization mode and acceleration mode, $P_{I O \text { static, }}$ and the power required to deliver a dynamic voltage pulse to transition between the two modes, $P_{l O}$ dynamic. The static power associated with maintaining a voltage gradient can be determined by combining Joule's Law and Ohm's law,

$$
P=\frac{V^{2}}{R}=\frac{V^{2} W t_{r}}{\rho L},
$$

where $V$ and $R$ are the voltage and electrical resistance, respectively, between ion optic electrodes, $W$ is the ion optic width, $t_{r}$ and $\rho$ are the thickness and resistivity of the resistive layer on the glass substrate, and $L$ is the distance between adjacent ion optic electrodes. The resistivity in thin films is frequently expressed in terms of sheet resistance, $R_{s}$, such that $\rho=R_{s} t_{r}$, yielding

$$
P=\frac{V^{2} W}{R_{s} L} .
$$

Doubling (12) to account for the parallel-plate construction yields the power for each section within the ion optics characterized by a voltage gradient, $P_{I O}$ siatic..section,

$$
P_{I O_{-} \text {static_section }}=\frac{2 V^{2} W}{R_{s} L},
$$

and is applicable to both ionization and acceleration modes. The power associated with operating in ionization mode, $P_{I O}$ ion, and acceleration, $P_{I O}$ accel, mode is found by summing all power contributions from each section and scaling by the duty cycle, such that

$$
P_{I O_{-} \text {static }}=\sum_{i=\text { section }} P_{1 O_{-} \text {ion }}+\sum_{i=\text { section }} P_{I O_{-} \text {accel }} .
$$

Given that the instrument will be operated in ionization mode for $5 \times 10^{-5} \mathrm{~s}$, the time for the heaviest resolvable ions to reach the detector, and pulsed in acceleration mode for only $1 \times 10^{-8} \mathrm{~s}$, the large disparity in duty cycles means that $P_{I O}$ ion $>P_{I O}$ accel, reducing $(14)$ to

$$
P_{I O_{-} \text {static }}=\sum_{i=\mathrm{sec} \text { tion }} P_{I O_{-} i o n} \text {. }
$$

The only ion optic section with a non-zero voltage gradient occurs between electrodes $31-46$, and is characterized by $V$ $=515 \mathrm{~V}, W=15 \mathrm{~mm}, L=7.5 \mathrm{~mm}$, and $R_{s}=1 \times 10^{11} \Omega$ square $^{-1}$ (as quoted by the manufacturer). Thus, $P_{I O}$ static $=1 \times$ $10^{-5} \mathrm{~W}$.

In order to determine the power required to transition between ionization mode and acceleration mode, it is useful to approximate adjacent linear thin film electrodes patterned on the resistive glass substrate surfaces as two parallel wires, with a wire radius, $r_{w}$, on the order of the electrode film thickness, $t_{f}$, separated by a distance, $L$, and running the entire ion optic width, $W$. The capacitance, $C$, between two parallel wires is then

$$
\left.C=\frac{\left(27.78 \times 10^{-12}\right) W}{\ln \left(L / r_{w}\right)} \quad \text { (in F }\right)^{8} \text {. }
$$

Given that the total energy, $U$, stored in this parallel wire system is 


$$
U=\frac{1}{2} C V_{p}^{2},
$$

where $V_{P}$ is the final voltage between the two wires, then the power required is

$$
P=\frac{d U}{d t}=C V_{P} \frac{d V_{P}}{d t} .
$$

where $t$ is time and $d V_{p} / d t$ is the maximum slew rate. Substituting (16) and doubling (18) to account for the parallelplate construction yields the power for each section within the ion optics characterized by dynamic voltage change between ionization and acceleration mode, $P_{I O}$ dynamic sectuon,

$$
P_{I O O_{\text {dynamic_section }}}=\left(\frac{\left(55.56 \times 10^{-12}\right) W}{\ln \left(L / r_{w^{\prime}}\right)}\right) V_{P}\left(\frac{d V_{P}}{d t}\right) \text {. }
$$

The power associated with this dynamic operation is found by summing all power contributions from each ion optic section such that

$$
P_{I O_{-} \text {dynamic }}=\sum_{i=\sec t i o n} P_{I O_{-} \text {dynamic_section }} \text {. }
$$

In the simulated Mini TOF-MS model, the ion optics are defined by three sections: electrodes $1-16$, electrodes $16-31$, electrodes $31-46$. The section between electrodes $1-16$ is characterized by $L=7.9 \mathrm{~mm}$ and $V_{P}=265 \mathrm{~V}$. The section between electrodes $16-31$ is characterized by $L=7.9 \mathrm{~mm}$ and $V_{P}=-265 \mathrm{~V}$. The section between electrodes $31-46$ is characterized by $L=7.5 \mathrm{~mm}$ and $V_{P}=-250 \mathrm{~V}$. All ion optic sections are characterized by $W=15 \mathrm{~mm}$ and $r_{w}=5 \times 10^{-4}$ $\mathrm{mm}$. Assuming a slew rate $\sim 50 \mathrm{~V} / \mathrm{ns}$ (equivalent to a $5 \mathrm{~ns}$ rise time) and scaling by the duty cycle, yields an average power, $P_{I O \text { aynamic }}$, of $4 \times 10^{-4} \mathrm{~W}$.

Thus the total power consumed by the ion optics, $P_{I O}$, being dominated by the dynamic power, is also $4 \times 10^{-4} \mathrm{~W}$.

The power required to maintain the voltage gradients across the five sections of the reflectron mass analyzer can be found in a manner analogous to that used to determine $P_{I O \text { static }}$ above. Table 3 lists the dimensional and electrical parameters for each reflectron section as given in the Mini TOF-MS model. All reflectron sections were characterized

\begin{tabular}{|c|c|c|c|c|c|}
\hline & $\begin{array}{c}\text { Reflectron } \\
\text { electrodes } 1-7\end{array}$ & $\begin{array}{c}\text { Reflectron } \\
\text { electrodes } 7-13\end{array}$ & $\begin{array}{l}\text { Reflectron } \\
\text { electrodes } 13-19\end{array}$ & $\begin{array}{l}\text { Reflectron } \\
\text { electrodes } 19-24\end{array}$ & $\begin{array}{l}\text { Reflectron } \\
\text { electrodes } 24.29\end{array}$ \\
\hline Wh & 30 & 30 & 30 & 25 & 25 \\
\hline Vow DU & 24.2 & 72.8 & 121.4 & 128.6 & 173 \\
\hline
\end{tabular}
by $W=80 \mathrm{~mm}$ and $R_{s}=1 \times 10^{11} \Omega$ square ${ }^{-1}$. Thus, the total power consumed by the reflectron mass analyzer, $P_{R e f}$, is $4 \mathrm{x}$ $10^{-6} \mathrm{~W}$.

Table 3: Spacing and voltage between adjacent reflectron mass analyzer electrodes.

In order to calculate the power demands of the MCP detector, it is useful to consider the electrical circuit shown in Figure 9. The average rate of ion impingement at the detector, $I_{i}$, generates a current within the MCP detector, $I_{M C^{C} P}$,

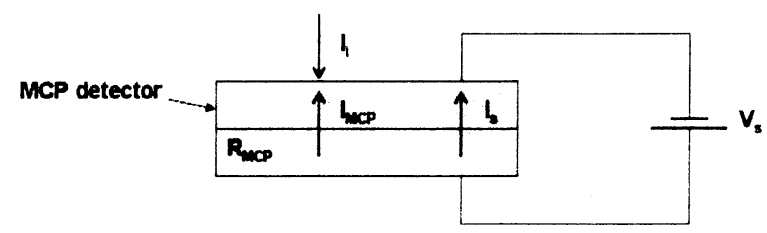

Fig. 9: Schematic electrical circuit of a MCP detector arranged in a chevron configuration. 
dependent upon the detector gain, $G$, such that

$$
I_{M P}=I_{1} G e .
$$

In order to ensure sufficient electrons to minimize detector dead time, the current supplied by the power supply, $I_{s}$, should be an order of magnitude greater than $I_{M(} P$, as in

$$
I_{s} \approx 10 I_{M(P} .
$$

Combining Joule's Law and Ohm's Law yields the total power required by the supply to operate the MCP detector, $P_{D e t}$,

$$
P_{\text {Det }}=V_{s} I_{s}=10 V_{s} I_{t} G e .
$$

Applying the Ideal Gas Law and evaluating the simulated Mini TOF-MS prototype sensitivity $\left(9 \times 10^{-5} \mathrm{cps} / \mathrm{molecule}\right.$ $\mathrm{N}_{2} / \mathrm{cc}$ for an $8 \mathrm{~mm}$ miniature TOF detector) at $300 \mathrm{~K}$ and $10^{-6}$ torr, yields an average rate of ion impingement rate, $I_{i}$, of 3 x $10^{6}$ ions/s. Experience with this miniature MCP detector through laboratory testing demonstrate $G=3 \times 10^{7} \mathrm{e}-/ \mathrm{s}$ at $V_{S}$ $=2050 \mathrm{~V}$. Thus, the total power required to operate the MCP detector is $0.2 \mathrm{~W}$. Application of Ohm's Law provides the resistance across the MCP detector plates of $1.7 \times 10^{7} \Omega$ given $I_{S}=1 \times 10^{-4} \mathrm{~A}$ and $V_{S}=2050 \mathrm{~V}$, which is within the manufacturer's product specifications.

Evaluating (9) results in the total power required to operate the proof-of-concept Mini TOF-MS prototype, $P_{\text {MiniTOF-MS, }}$ which is $0.3 \mathrm{~W}$. The power consumption is dominated by MCP detector needs.

The same methodology can be applied to a Mini TOF-MS design that features an extended ion source and MCP detector with a multiplicative factor of $M=25$. In this case, application of the Ideal Gas Law to the calculated Mini TOF-MS sensitivity $\left(3 \times 10^{-3} \mathrm{cps} /\right.$ molecule $\left.\mathrm{N}_{2} / \mathrm{cc}\right)$ at $300 \mathrm{~K}$ and $10^{-6}$ torr, results in an ion impingement rate, $I_{i}$, of $2 \times 10^{8}$ ions $/ \mathrm{s}$. To handle this increased ion flux, a larger, single strip MCP detector with different operating parameters $\left(V_{S}=2400 \mathrm{~V}\right.$, $G=4 \times 10^{6} \mathrm{e}-/ \mathrm{s}, R_{M C^{\prime} P}=4 \times 10^{6} \Omega$ ) can be used ${ }^{\ddagger \ddagger}$. The total power required to operate an Mini TOF-MS design modified for high sensitivity is found by evaluating (9) and is $1.3 \mathrm{~W}$, which represents over an order of magnitude improvement in power consumption than current state-of-the-art mass spectrometers such as the SAM QMS (14.5 W for thermionic emission ion source, quadrupole mass analyzer, and detector components). These results establish the theoretical basis for resource-friendly, low power operation of the Mini TOF-MS in planetary science applications.

\section{HIGHER PERFORMANCE DESIGNS}

We have also investigated higher performance TOF-MS designs in an effort to meet science objectives demanding higher mass resolution. One approach towards higher mass resolution is to extend the total ion flight length. This can be accomplished by again taking advantage of the parallel plate Mini TOF-MS geometry to produce a stacked double reflectron TOF-MS as shown schematically in Figure 10a. An integrated model based upon this general design was modeled and its simulated performance was characterized. The resultant spectrum of two chemical species of

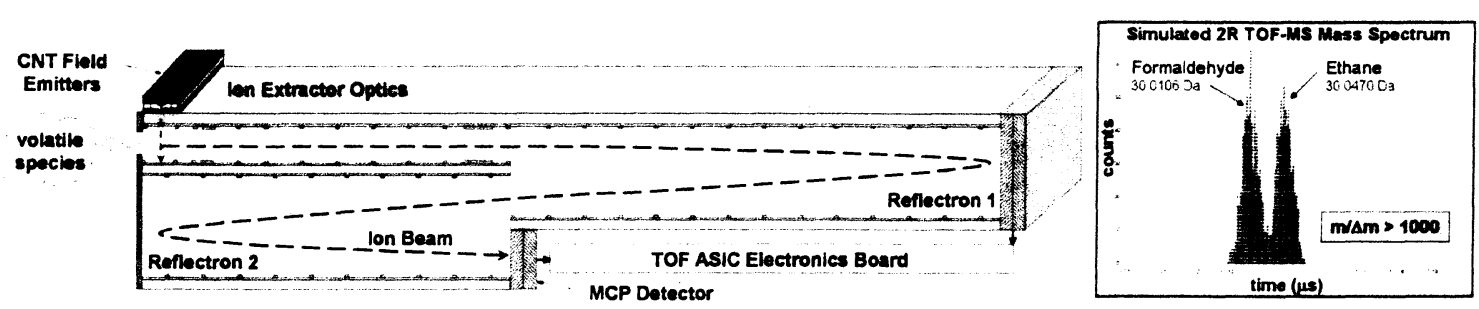

Fig. 10: (a) Schematic cross section of a double reflectron Mini TOF-MS design for achieving higher mass resolution. (b) Simulated mass spectrum of formaldehyde and ethane demonstrates unit mass resolution at $1000 \mathrm{Da}$.

\footnotetext{
${ }^{\ddagger \ddagger}$ For example, a custom-cut, $75 \mathrm{~mm}$ wide chevron MCP detector with extended dynamic range, ADP 3075MA 32/25/8

D EDR 60:1, Photonis USA, Inc., www.photonis.com.
} 
$\because$ astrobiological interest (formaldehyde and ethane) is shown in Figure 10b and demonstrates a mass resolution of 1000 within a core volume of $1130 \mathrm{~cm}^{3}$. The price of greater resolution however is decreased sensitivity. The transmission efficiency in the double reflectron TOF-MS configuration was $1 \%$ which corresponds to an instrument sensitivity of $4 \mathrm{x}$ $10^{-4} \mathrm{cps} /$ molecule $\mathrm{N}_{2} / \mathrm{cc}$ for an ion source extended by a multiplicative factor of 25 . Despite the lower sensitivity, such a device would still provide significant capability for a space flight mass spectrometer.

\section{CONCLUSIONS AND FUTURE DIRECTIONS}

We have described a design of a miniature TOF-MS for astrobiological and planetary science that utilizes nano- and micro-fabricated components and have characterized its high performance attributes via SIMION electrodynamic simulations. The modeled Mini TOF-MS design has been shown to exhibit a mass resolution of 600 and a sensitivity of $7 \times 10^{-5} \mathrm{cps} /$ molecule $\mathrm{N}_{2} / \mathrm{cc}$ within a small volume of $840 \mathrm{~cm}^{3}$ for core elements (i.e. ion source, reflectron mass analyzer, and detector) and lays the theoretical foundation for a sound proof-of-concept prototype build. Reasonable extrapolations suggest that attainment of higher sensitivity on the order of $5 \times 10^{-3} \mathrm{cps} /$ molecule $\mathrm{N}_{2} / \mathrm{cc}$ with the Mini TOF-MS architecture is possible through extension of the ion source without sacrificing mass resolution or instrument volume. Higher performance modifications to the basic Mini TOF-MS design such as incorporating a double reflectron configuration have also been investigated with electrodynamic simulations and demonstrate achievement of mass resolutions of $>1000$, albeit with lower sensitivity $\left(3 \times 10^{-4} \mathrm{cps} / \mathrm{molecule}_{2} / \mathrm{cc}\right)$ and a slightly larger core volume (1130 $\mathrm{cm}^{3}$ ).

Future efforts will focus on refining the Mini TOF-MS performance simulations by incorporating realistic voltage pulse rise times and pulse widths as well as electron beam space charge effects in order to more accurately quantify the impact on instrument resolution and sensitivity. We are currently engaged in Mini TOF-MS component microfabrication and assembly in preparation for laboratory performance testing of the integrated prototype.

\section{ACKNOWLEDGEMENTS}

The authors are very grateful to Mehdi Benna for imparting valuable SIMION expertise, to Hasso Niemann for many fruitful discussions, to Jason Dworkin, and Danny Glavin for astrobiological insight, and Dan Harpold for help with SAM QMS comparisons. This work has been supported by the NASA ROSES Astrobiological Science and Technology Instrument Development [ASTID04-0000-0067] and by the NASA Goddard Space Flight Center Internal Research and Development [IRAD] program.

\section{REFERENCES}

\footnotetext{
1 NP-2006-02-423-HQ, www.nasa.gov/pdf/142302main_2006_NASA_Strategic_Plan.pdf

NP-2004-01-334-HQ, http://www.nasa.gov/mission_pages/exploration/main/index.html, The Vision

R.J. Cotter, Time-of-Flight Mass Spectrometry: Instrumentation and Applications in Biological Research, ACS

Professional Reference Books, Washington, DC, 1997.

4 Jordan TOF Products Inc., Time of Flight / TOF Mass Spectrometer Tutorial, http://www.rmjordan.com/Resources/Tutorial.pdf.

J. Gross, Mass Spectrometry, Springer-Verlag, Berlin, 2004.

6 T.J. Cornish and R.J. Cotter, "A Curved Field Reflectron Time-of-Flight Mass Spectrometer for the Simultaneous

Focusing of Metastable Product lons," Rapid Communications in Mass Spectrometry, 8, 781-785 (1994).

7 National Institute of Standards and Technology Physics Laboratory, Physical Reference Data, Electron-Impact

Cross Sections for Ionization and Excitation, http://physics.nist.gov/PhysRefData/lonization/Xsection.html.

8 C.R. Paul, Introduction to Electromagnetic Compatibility, Wiley-Interscience, pg. 309, 2006.
} 